\title{
Dosage de I'ATP microbien pour l'estimation de la flore totale du lait cru
}

\author{
A Branger ${ }^{1}$, I Cuvillier ${ }^{1}$, L Rabhi² \\ ${ }^{1}$ ENILBIO, BP 49, 39801 Poligny Cedex; \\ ${ }^{2}$ Lumac Perstorp, 1, rue Jean-Carasso, 95870 Bezons, France
}

(Recu le 18 janvier 1995 ; accepté le 16 mars 1995)

\begin{abstract}
Résumé - La technique de dosage d'ATP que nous avons étudiée utilise la filtration de $300 \mu \mathrm{l}$ de lait cru à l'intérieur d'un bloc chauffant à $40^{\circ} \mathrm{C}$. La membrane supportant les micro-organismes sert en totalité pour le dosage de l'ATP microbien. Après analyse en double de 100 échantillons de lait, de producteur ou de citerne, par la méthode de référence en boîte de Pétri et le dosage d'ATP, nous avons calculé les paramètres analytiques de précision. La gamme de concentration microbienne était de $310^{3}$ à $10^{7}$ micro-organismes par ml. L'écart type de répétabilité est de 0,108 log ufc/ml. La justesse exprimée par la précision d'estimation est de 0,508 , et l'équation de régression : $y=1,6 x-0,47$. On constate que la justesse est bonne mais qu'un calibrage est nécessaire. Le travail réalisé ici montre que le dosage d'ATP a fait de réels progrès dans la quantification des populations microbiennes du lait cru en montrant des valeurs pour les paramètres de précision, proches, voire meilleures que pour les techniques habituellement utilisées pour le dénombrement microbien dans le lait cru.
\end{abstract}

\section{ATP/ flore totale / lait cru / justesse / répétabilité}

Summary - Use of an ATP assay technique to determine the microbial quality of raw milk. The assayed ATP level determination technique uses the filtration of $300 \mu$ l of raw milk inside a $40^{\circ} \mathrm{C}$ heating system. The membrane holding the microorganisms is wholly used to determine the bacterial ATP levels. After duplicate analysis of 100 farm or tank milk samples by the reference method in Petri dishes and ATP determination, we have calculated the analytical precision parameters. The microbial concentration ranged from $310^{3}$ to $10^{7}$ microorganisms $/ \mathrm{ml}$. The repeatability standard deviation is $0.108 \mathrm{log} \mathrm{cfu} / \mathrm{ml}$. The accuracy is 0.508 and the regression equation : $y=1.6 x-0.47$. It can be seen that the accuracy is good but that calibration is necessary. This study shows that the determination of ATP levels has greatly improved the counting of raw milk microorganims by showing values for precision parameters close and even better than with the commonly used techniques for raw milk microbial counts. 


\section{INTRODUCTION}

L'ATP (adénosine triphosphate), est un trinucléotide intracellulaire, en majeure partie responsable des transferts énergétiques du métabolisme.

Cette molécule existe dans toutes les cellules vivantes, et son dosage peut être utilisé pour une quantification de biomasse, en particulier pour l'estimation des populations microbiennes présentes dans les produits alimentaires.

Dans les produits laitiers, cette méthode a été largement étudiée, en particulier pour le dénombrement rapide des cellules somatiques (Bossuyt, 1978), pour l'estimation de la population microbienne du lait cru (Britz et al, 1980 ; Bossuyt, 1981 et 1982 ; Theron et al, 1986a etb; Botha etal, 1986; Langeveld et Van der Waals, 1988; Van Crombrugge etal, 1989; Rongvaux-Gaïda et Piton 1991 ; Rongvaux Gaïda et al, 1991) pour la détection de la contamination de lait et de crème pasteurisés (Waes et Bossuyt, 1981 ; Griffiths et al, 1984) ou encore pour détecter la non stérilité des laits UHT (Waes et al, 1984).

Le dosage de l'ATP à partir des micro-organismes présents dans le lait cru nécessite plusieurs étapes : élimination de l'ATP non microbien, extraction de I'ATP microbien et dosage enzymatique avec mesure de la luminescence produite par la réaction.

La première phase est la plus délicate. Elle permet l'élimination du bruit de fond important dû à l'ATP libre du lait cru et à celui quí est contenu dans les cellules somatiques. Pour cela, plusieurs techniques ont été étudiées : I'ATP platform ou ATP-P (Bossuyt, 1982) le $\triangle A T P$ test (Langeveld et Van der Waals, 1988; Rongvaux-Gaïda et Piton, 1991), ou l'ATP-F test (Van Crombrugge et al, 1989; Rongvaux-Gaïda et Piton, 1991).

De ces 3 techniques, comparées par Rongvaux-Gaĩda et Piton (1991), l'ATP-F test semble être la mieux adaptée à l'estimation de la flore totale du lait cru.

Nous avons repris cette technique, après la mise au point par la station laitière de Melle en Belgique (Reybroeck et Schram, 1994), d'un nouveau kit de réactif, le Raw Milk Microbial Kit (Lumac, référence 9285-1 ; fournisseur : Perstorp Analytical, BP 13, 95871 Bezons Cedex, France,) auquel est associé un bloc de filtration chauffant (Lumac Biofiltration System, réf 9133-3). Le but est d'observer d'éventuelles améliorations des paramètres de précision (justesse et répétabilité), par rapport aux études précédentes.

\section{MATÉRIEL ET MÉTHODE}

\section{Échantillons utilisés}

Nous avons analysé 100 échantillons de lait cru, dont 12 laits de mélange et 88 laits de producteurs. Les niveaux de contamination couvraient la gamme $310^{3}$ à $10^{7} \mathrm{ufc} / \mathrm{ml}$.

Après le prélèvement, les échantillons ont été conservés à $4^{\circ} \mathrm{C}$ au réfrigérateur jusqu'au moment de l'analyse.

\section{Technique de référence utilisée}

Les échantillons ont été analysés par la technique de référence par dilutions manuelles, norme FIL 100B (Anonyme, 1991) après une agitation standard de 25 retournements d'une amplitude de 25 à $30 \mathrm{~cm}$ en 7 à $10 \mathrm{~s}$ (Anonyme, 1978). Chaque échantillon a été analysé en double, et les comptages ont été réalisés à partir de boîtes contenant de 30 à 300 colonies.

\section{Dosage de I'ATP}

Le protocole proposé diffère essentiellement de celui utilisé par Rongvaux-Gaïda et Piton (1991) par l'utilisation d'un système de filtration automatique chauffant, des volumes de réactifs et échantillons differents, et par le fait que le dosage se fait sur la totalité de la membrane filtrante.

Les étapes de l'analyse sont les suivantes: après montage d'une membrane de nylon de $8,3 \mathrm{~mm}$ de diamètre et de $0,8 \mathrm{~mm}$ de porosité 
dans le système de filtration, on apporte dans l'entonnoir $300 \mu \mathrm{l}$ de L-NRS, extractant de l'ATP somatique préchauffé à $40^{\circ} \mathrm{C}$ et $300 \mu \mathrm{l}$ de lait cru ayant subi l'agitation standard. L'ensemble du bloc de filtration est chauffé à $40^{\circ} \mathrm{C}$. Après 4 min d'incubation pendant lesquelles l'ATP des cellules somatiques est libéré, on filtre sous une dépression de $93,3 \mathrm{kPa}(700 \mathrm{~mm} \mathrm{Hg})$. La membrane est rincée avec $600 \mu \mathrm{l}$ de liquide de Ringer au $1 / 4$ lui aussi préchauffé à $40^{\circ} \mathrm{C}$.

La membrane supportant les micro-organismes et débarrassée de l'ATP somatique est transférée dans un tube à fond plat qui est placé dans le luminomètre (Biocounter TM M 2500) ; $200 \mu$ l d'extractant bactérien (NRM) sont ajoutés automatiquement, puis après $30 \mathrm{~s}$ d'action, une injection automatique de Lumit - QM permet le déclenchement de la réaction. La lumière émise est mesurée à partir d'une intégration de $10 \mathrm{~s}$. Le résultat est alors affiché en URL (unités relatives de lumière). Entre 2 analyses, le bloc de filtration est rincé par 2 fois avec $600 \mu$ d'eau distillée stérile exempte d'ATP. Chaque échantillon a également été analysé en double par cette technique.

\section{Calculs statistiques}

Ils ont été réalisés selon les directives de la norme AFNOR V-03-110 (Anonyme, 1993) après transformation des résultats URL et comptages microbiens, en logarithmes en base 10 .

\section{RÉSULTATS ET DISCUSSION}

\section{Étude de la répétabilité}

Le tableau I regroupe les paramètres de répétabilité des 2 techniques utilisées.
La valeur de variance de répétabilité obtenue pour la méthode de référence est inférieure au seuil couramment admissible qui est de 0,012 (Brazis, 1991). La valeur obtenue pour la mesure d'ATP ne diffère pas significativement de la précédente au seuil de $5 \%$ (test $F$ de comparaison des variances) et est beaucoup plus faible que celle obtenue par Rongvaux-Gaïda et Piton (1991) est qui était de 0,025 .

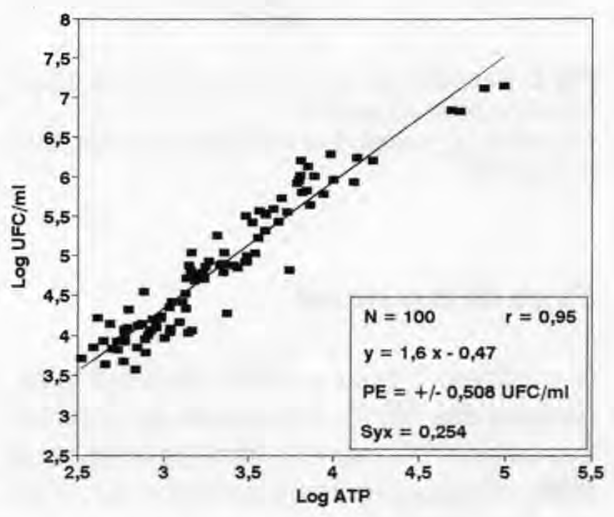

Fig 1. Étude de la justesse de la technique ATP par rapport à la référence en boîte de Pétri. $\mathrm{N}$ : nombre d'échantillons ; $r$ : coefficient de corrélation ; PE : précision d'estimation ; PE : $\pm 2 \mathrm{Sy}, \mathrm{x}$; Sy, $x$ : écart type résiduel.

Accuracy study between ATP technique and reference method. $N$ : number of samples; $r$ : correlation coefficient; PE : \pm 2 Sy, $x ;$ Sy, $x$ : residual standard deviation.

Tableau I. Valeurs de répétabilité pour la méthode de référence et le dosage ATP. Pour ce dernier, les valeurs ont été transformées en log ufc grâce à l'équation de la droite de régression. Repeatability for the reference method and ATP measure. The values of ATP were transformed in $\log$ ufc with the equation of the regression line.

\begin{tabular}{llcc}
\hline Méthodes & \multicolumn{1}{c}{$S r^{2}$} & Sr (en log ufc/ml) & $r$ (en log ufc/ml) \\
\hline Gélose PCA $30^{\circ} \mathrm{C}$ & 0,0074 & 0,0861 & 0,241 \\
ATP Lumac & 0,017 & 0,108 & 0,302 \\
\hline
\end{tabular}




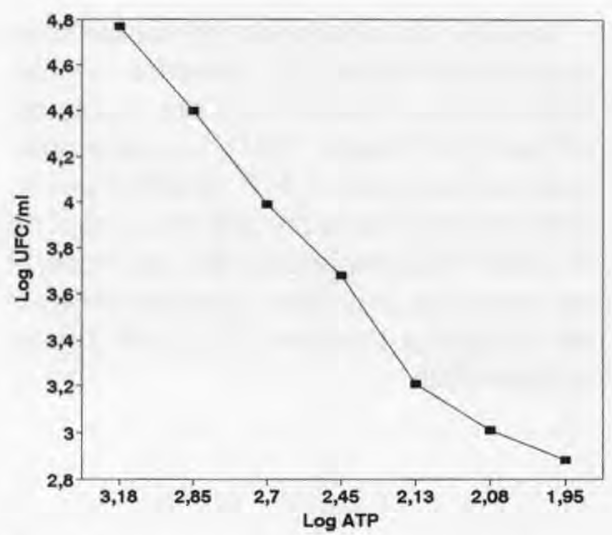

Fig 2. Exemple de recherche du seuil de détermination pour un lait cru.

Example of research of the determination level in raw milk.

\section{Étude de la justesse}

Sur la figure 1, nous pouvons observer la répartition des points, les valeurs de paramètres caractéristiques et le tracé de la droite de régression qui expriment la relation qui existe entre les 2 techniques.

On peut constater que l'ajustement de la droite n'est pas correct. La pente et l'ordonnée à l'origine sont significativement différentes respectivement de 1 et de 0 . La technique nécessite donc un calibrage.

La précision d'estimation, calculée à partir de l'écart type résiduel est correcte ( $\pm 0,508$ log ufc, ce qui se rapproche de l'écart couramment admis de $\pm 0,5$ log ufc, et des valeurs trouvées pour les techniques microscopiques en épifluorescence : $\pm 0,480$ log ufc pour la technique DEFT (Dasen et al, 1987) et $\pm 0,580$ log ufc pour le Bactoscan (Grappin et al, 1985).

\section{Seuil de détermination}

Nous avons tenté d'estimer le seuil de détermination en diluant 20 échantillons de laits crus différents dans du lait UHT stérile et en mesurant le niveau d'ATP bactérien dans chaque dilution.

La figure 2 présente un exemple de résultat. Nous observons dans la plupart des cas, une évolution du taux d'ATP en fonction du nombre de micro-organismes, avec 2 pentes. Le point d'inflexion se situe entre 3 et $3,5 \mathrm{log}$ ufc par $\mathrm{ml}$. On peut donc retenir comme seuil de détermination la valeur de 3,5 log ufc par ml, c'est-à-dire environ $3,210^{3}$ ufc par ml.

\section{CONCLUSION}

Le nouveau kit Lumac, le Raw Milk Microbial Kit pour le dénombrement de la flore totale dans le lait cru par le dosage de l'ATP microbien, a permis une nette amélioration des caractéristiques analytiques de cette technique.

Le protocole nouvellement développé, comprenant l'utilisation d'un système automatique de filtration chauffant, et l'utilisation directe de la membrane, a permis d'amener cette technique au niveau des techniques officielles actuelles pour le dénombrement microbiologique pour le paiement du lait à la qualité.

Pour cela, il est nécessaire d'utiliser une équation de calibrage qui peut être celle de la régression donnée par l'étude de la justesse. Enfin, la diminution des volumes des réactifs a permis également la réduction des coûts de l'analyse.

\section{RÉFÉRENCES}

Anonyme (1978) Standard methods for the examination of dairy products, 14 th edn. American Public Health Association, New York

Anonyme (1991) Lait et produits laitiers. Dénombrement des micro-organismes. Comptage des colonies à $30^{\circ} \mathrm{C}$. Norme FIL $100 \mathrm{~B}$

Anonyme (1993) Analyse des produits alimentaires. Protocole d'évaluation d'une méthode 
d'analyse quantitative par rapport à une méthode de référence. Norme AFNOR V03-110

Bossuyt R (1978) Usefulness of an ATP assay technique in evaluating the somatic cell content of milk. Milchwissenschaft 33, 11-13

Bossuyt R (1981) Determination of bacteriological quality of raw milk by an ATP assay technique. Milchwissenschaft 36, 257-260

Bossuyt R (1982) A 5-minute ATP platform test for judging the bacteriological quality of raw milk. Neth Milk Dairy J 36, 355-364

Botha WC, Lück H, Jooste PJ (1986) Determination of bacterial ATP in milk. The influence of adenosine triphosphate - hydrolysing enzymes from somatic cells and Pseudomonas fluorescens. J Food Prot 49, 822-825

Brazis AR (1991) Methods for estimating colony forming units. Plate count method. Bull Int Dairy Fed 256, 4-8

Britz JJ, Bezuidenhout JJ, Dreyer JM, Steyn PL (1980) Use of adenosine triphosphate as an indicator of the microbial counts in milk. $S$ Afr J Dairy Technol 12, 89-91

Dasen A, Piton C, Grappin R, Guerry P (1987) Évaluation de la technique DEFT associée à un comptage visuel ou à un comptage par analyseur d'images pour la numération de la flore totale du lait cru. Lait 67, 77-95

Grappin R, Dasen A, Favennec P (1985) Numération automatique et rapide des bactéries du lait cru à l'aide du Bacto-Scan. Lait 65, 123147

Griffiths MW, Phillips JD, Muir DD (1984). Methods for rapid detection of post pasteurization contamination in cream. J Soc Dairy Technol 37, 22-26

Langeveld LPM, Van der Waals CB (1988). The ATP platform test, the $\triangle$ ATP test and the direct microscopic count procedure as methods of estimating the microbial quality of raw milk. Neth Milk Dairy J 42, 173-182

Reybroeck W, Schram E (1994) Integrated filtration method for testing the bacteriological quality of raw milk by ATP bioluminescence. $I n$ : Bioluminescence and chemiluminescence; fundamentals and applied aspects. Proc 8th Int Biolum and Chemilum Symp (AK Campbell, LJ Kricka, PE Stanley, eds). John Wiley \& Sons, Chichester, 482-485

Rongvaux-Gaïda D, Piton C (1991) Comparaison de 3 méthodes de dosage de l'ATP par bioluminescence pour l'estimation de la qualité bactériologique du lait cru. Lait 71, 483-491

Rongvaux-Gaïda D, Piton-Malleret C, Dasen A (1991) Dosage de l'ATP microbien du lait cru par l'appareil Bacto Foss. Lait 71, 575-587

Theron DP, Prior BA, Lategan PM (1986a) Determination of bacterial ATP levels in raw milk: selectivity of non-bacterial ATP hydrolysis. $J$ Food Prot 49, 4-7

Theron DP, Prior BA, Lategan PM (1986b) Sensitivity and precision of bioluminescent techniques for enumeration of bacteria in skim milk. $J$ Food Prot 49, 8-11

Van Crombrugge J, Waes G, Reybroeck W (1989) The ATP-F test for estimation of bacteriological quality of raw milk. Neth Milk Dairy J 43, 347-354

Waes G, Bossuyt R (1981) A rapid method to detect post contamination in pasteurized milk. Milchwissenschaft 36, 548-552

Waes G, Bossuyt R, Mottar J (1984) A rapid method for the detection of non-sterile UHT milk by the determination of the bacterial ATP. Milchwissenschaft 39, 707-711 